\title{
Artifacts Reduction in Image Rendering of the Focused Plenoptic Camera \\ Peng Liư ${ }^{1}$, Rumin Zhang ${ }^{2}$
}

School of Electronic and Information Engineering, Beijing University of Aeronautics \& Astronautics, No.37 Xueyuan Road, Haidian Distract,Beijing,China

State Key Laboratory of Wireless Mobile Communications, China Academy of Telecommunications Technology (CATT) ,Beijing,China

715248894@qq.com, liudijun@leadcoretech.com

Keywords: plenoptic camera;micro lens array; artifacts reducing; digital refocusing; depth of field

\begin{abstract}
Plenoptic camera provides us with the capability of refocusing photograph and extending the depth of field(DOF) after exposure. But when we render image patch by patch, the output image is rendered with visible artifacts because of mismatch of the adjacent patch boundaries. The paper introduce a novel image rendering algorithm based on the focused plenoptic camera. The algorithm extracts the depth information from the raw light field data, and then render the result image patch by patch based on the depth map. The algorithm can export an image setting in a desirable focus. It can also extend the depth of field to the case that the whole scene in the image is in focus. And from the simulation, it can be learnt that comparing with the traditional algorithm, the artifacts in the rendering result are suppressed significantly to a sightless level.
\end{abstract}

\section{Introduction}

Plenoptic camera came out in recent years. It implements a high efficient, portable and low cost device to record both spatial and directional information of the scene. With the captured light field data, it is to set the focus and extend the depth of field when the exposure is over. Fourier slice is proposed to render refocus result in high efficiency. But it render only one pixel per sub image. So the resolution of the image rendered from plenoptic camera is as the number of the micro lenses in the array.

A novel structure of plenoptic camera was presented later by A.Lumsdaine and T.Geogiev. The micro lenses are carefully set to focus on the image formed by the main lens. So it is called focused plenoptic camera or plenoptic camera 2.0. It trades directional information for the spatial resolution of the light field information. Thus, the spatial resolution of focused plenoptic camera increases in the refocus result. However, it may lead to visible artifact due to lack of directional information.

Based on the model of focused plenoptic camera, the paper illustrates the relationship between the light field samples and the target rendering image, and introduces a novel algorithm to build an digital refocus and extended DOF image. The depth map of the scene is needed in the algorithm and it will be extracted by finding the correspondence between the sub images of adjacent micro lenses. With the assistant of new algorithm in the paper, when we render an refocus image from patch to patch corresponding to the each sub image, we will get a continuous transition at the joint of adjacent rendered patches. The spatial resolution of the final output picture is decided by the depth map of the scene.

\section{Rendering Image with a focused Plenoptic Camera}

\section{A. Focused Plenoptic Camera}

The structure of focused plenoptic camera proposed by A.Lumsdaine and T.Geogiev is as described in Fig. 1. It consists of a main lens and a micro lens array positioned close to the sensor. Each micro lens serves as a micro camera that samples the image of the main lens 
Micro lenses implement ray separators. Their image circles divide the sensor into sub images. The coordinate of one sub image $(m, n)$ combining with the pixel $(s, t)$ in this sub image give us the radiance corresponding to a definite direction and position. For all the sub images and pixels, the light field in the camera can be established as $I(s, t, m, n)$. In a practical plenoptic system, the micro lens is square. For simplicity, the following analysis only takes the $(s, m)$ dimension into consideration. For the $(n, t)$ dimension it has a similar conclusion.

The light field data $I(s, m)$ is read from the sensor data $I_{s}(x)$. Thus it has:

$I(s, m)=I_{s}(m * N x+s)$

where $N x$ is the resolution of each sub image. It has $N x=d / \delta$. Here d is the size of micro lens and $\delta$ is the diameter of the pixel on the sensor.

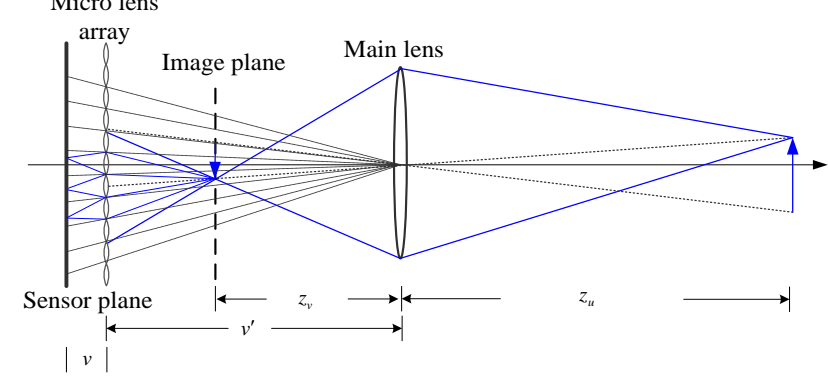

Figure 1. Structure of focused plenoptic camera

It is well known that the connection lines between the optic center of the main lens and the edge of the micro lenses divide the image of the main lens into $N X$ patches. It is the same as the number of micro lenses. And our algorithm will render from patch to patch on the different image planes. To render an artifacts suppressed result, the depth map corresponding to each image plane $Z_{m}(0 \leq m<N X)$ is necessary. A approach which extracts depth map from multi-views is proposed as follow.

\section{B. Depth Map Estimation}

Depth estimation can be considered as a minimization of the matching error of the images formed by neighbor micro lenses. For an arbitrary sub image $I(s, m)$ and its neighborhood, it has:

$$
\Phi\left(\Delta_{m}\right)=\frac{1}{W} \sum_{i}\left\|I\left(\Delta_{m}+i, m\right)-I_{s}(i, m+1)\right\|
$$

$\Delta_{m}$ is the disparity of pixels between adjacent sub images as in the Fig. 2. And $\mathrm{W}$ is number of matching pixels.

We need to search for the optimal $\Delta_{m}$ when the matching error $\Phi\left(\Delta_{m}\right)$ gets its minimum. And the map of depth can be calculated as:

$$
z_{m}=v^{\prime}-\frac{d}{\Delta_{m} \delta} v, 0 \leq m<N_{x}
$$




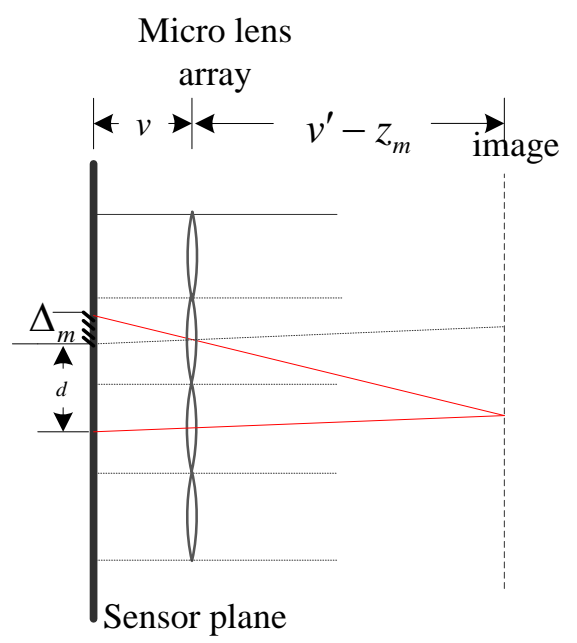

Figure 2. Depth map is extracted from the relation of neighbor sub images.

\section{Rendering Algorithm}

The proposed algorithm will render an image at specified F-number $N_{F}$ and refocus plane that has a distance of $\alpha v^{\prime}$. We will deduce the relationship between an arbitrary patch $\mathrm{m}$ and the light field $I(s, m)$. For an pixel $\mathrm{Z}(\mathrm{s})$ on the rendering patch $\mathrm{m}$, the light projection of an arbitrary point $\mathrm{Z}$ (s) to the micro lens array overlays a certain number of micro lenses, whose sub images offer effective information to the rendering patch. And it can be known that the diameter of this projection is decided by the specified photographing aperture size $N_{F}$ and depth of the patch $Z_{m}$. The diameter(number of micro lenses) can be calculated:

$$
L=\left[\frac{v^{\prime}-z_{m}}{z_{m}} \cdot \frac{F / N_{F}}{d}\right]
$$

[ ] is the rounding symbol. As it is shown in Fig.3, sub images of the micro lenses that lay within this range have effective information of $\mathrm{Z}(\mathrm{s})$. We consider them as the effective sub images of patch $\mathrm{m}$. And the radiance of $\mathrm{Z}(\mathrm{s})$ is the weighted average of all the corresponding red pixels of $\mathrm{Z}(\mathrm{s})$ on the sensor. Under the Lambertian assumption that the object produces redundant radiance in different angle, we can figure that all corresponding pixels of $\mathrm{Z}(\mathrm{s})$ on the sensor contribute the same to the radiance of $\mathrm{Z}(\mathrm{s})$. Thus all weights are equal.

From Fig.4, we can see the pixels underlined by red on the sensor contribute information to the patch $\mathrm{m}$, while those underlined by blue contribute to the patch $\mathrm{m}+1$. In each effective sub image, the number of effective pixels is:

$$
P\left(z_{m}\right)=\frac{v}{v^{\prime}-z_{m}} \cdot \frac{z_{m}}{v^{\prime}} \cdot \frac{d}{\delta}
$$

$P\left(z_{m}\right)$ is the function of the depth of the patch $m$. Thus different patch has different rendered resolution on its depth.

To get the final output, it is necessary to project all the patches from their depth to the reference plane. The reference plane is selected as the refocus plane, which is decided by the refocus factor $\alpha$. We scale the patch m by the resolution of $P\left(\alpha v^{\prime}\right)$ on the reference plane as shown in Fig. 4 . And the rendering result is the stitching of all the scaling patches. 


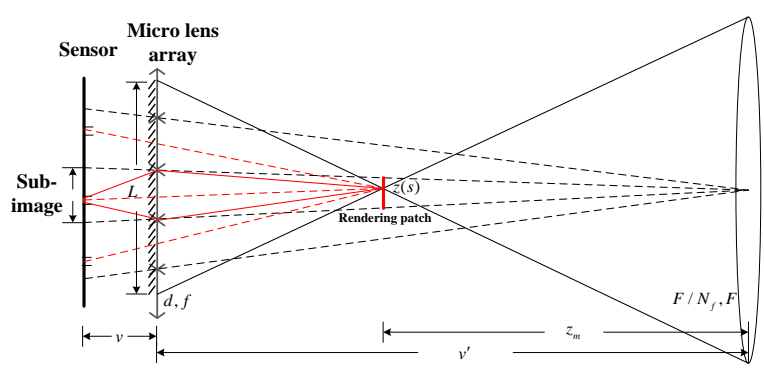

Figure 3. Repetitions of the rendering pixel on the sensor

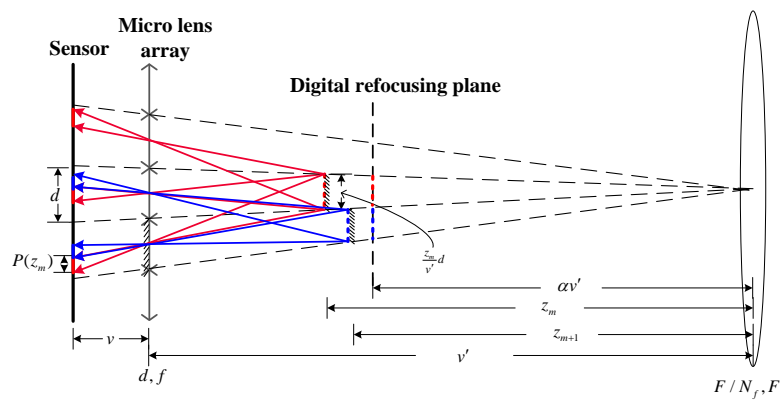

Figure 4. $\quad$ Rendering algorithm based on the depth map

\section{Experimental Results}

\section{Implementation}

Our Algorithm can perform as following 4 steps:

- The 4D light field of the scene is established from the sensor data.

- The depth map for all the patches to be rendered is estimated from the light field data.

- Every patches is rendered according to the depth map. They have different resolution due to different depth. Aperture factor $N_{F}$ is specified to decide the repetition of a rendering pixel on the sensor. It controls the DOF of the result.

- Project all the patches to the reference plane. The reference plane is selected as the specified refocus plane by the factor $\alpha$. The final refocusing result is the stitching of all the projections of rendered patches on the reference plane.

\section{E. Simulation Example}

Our simulation is done with the pre-processed light field data captured with a prototype of focused plenoptic camera by T. Georgiev. Fig. 5 is the raw light field image, which is composed of an array of sub images(96*72). Each micro lens maps part of the image of the main lens onto the sensor plane. Sub images are formed behind the micro lenses and have a resolution of $75 * 75$. If we zoom in and examine the difference between the adjacent sub images, we can clearly find a parallax shift.

Figure 5. Raw plenoptic data captured by focused plenoptic camera 
Fig. 6 indicates the depth map of the scene. We extract it by finding the parallax of the sub images. The light part on the picture means shallow depth where the dark part means deep depth. The resolution of depth map is as much as the number of number of rendering patches.

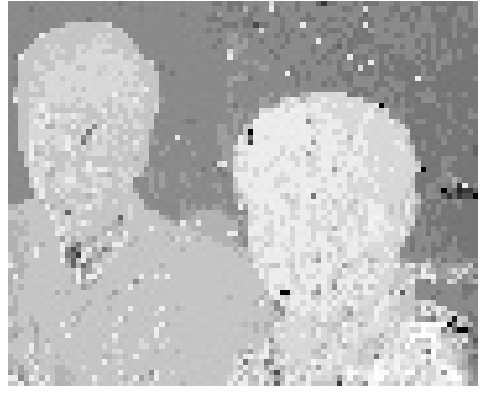

Figure 6. Depth map extracted from the plenoptic data

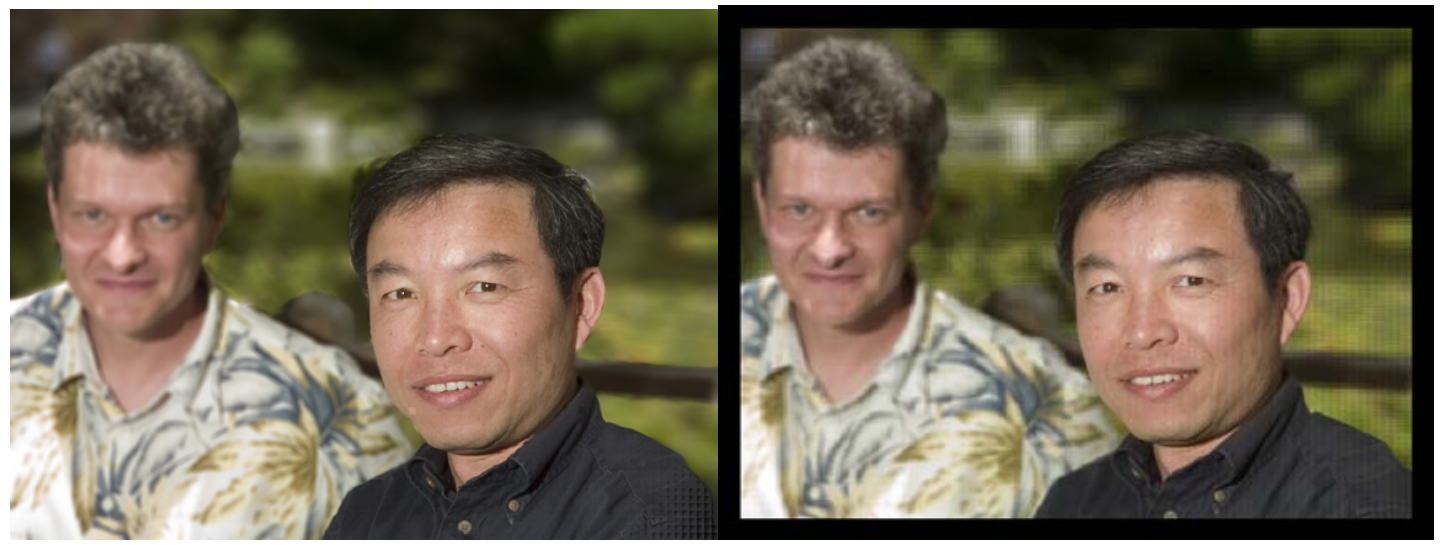

Figure 7. Simulation example (a) Reconstruction of refocus image with the proposed algrotihm (b) without depth map

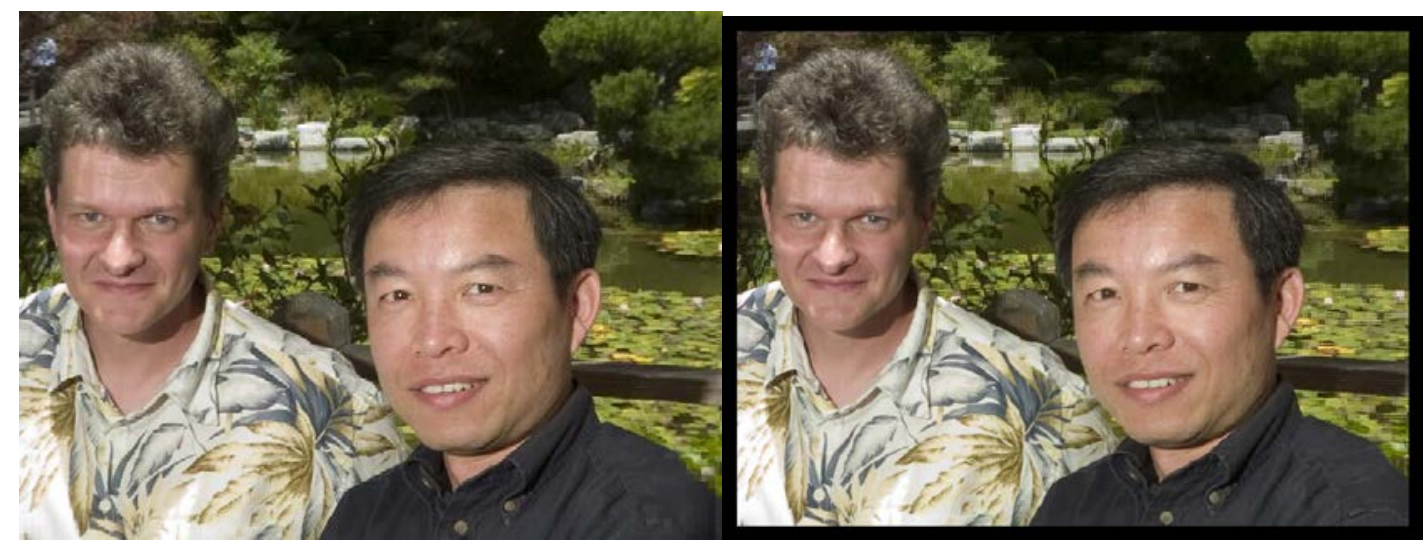

Figure 8. Simulation example (a) Reconstruction of all-in-focus image with the proposed algrotihm (b) without depth map

Fig. 7(a) shows the rendered image with the algorithm in the text. The refocus factor $\alpha$ is carefully set to refocus the image at the face of the person in the front. Fig. 7(b) shows the result rendered by algorithm without depth estimation. From the comparison we can learn that the result in Fig. 7(b) has visible artifacts especial in the area out of focus because of sparse . And in Fig. 7(a), the background is smoothly blurring and artifacts are suppressed obviously.

Fig 10(a) shows rendered image that the F-number $N_{F}$ is set to its maximum. And the depth of field is extended to maximum. The image is all-in-focus from the wood in the background to the people in the foreground. Fig 10(b) is the all-focus result rendered by algorithm without depth estimation. It is well known that the artifacts are sightless after using the proposed algorithm when we render an image that is all focus. 


\section{Conclusions}

Reconstruction resolution is one of the main limitations put on the application of plenoptic camera. When we use focused plenoptic camera to trade directional resolution for high rendering resolution, it may bring about artifacts in the final output due to lack of directional information. The paper first discussed the structure of focused plenoptic camera proposed by Georgiev, T. and Lumsdaine, A. Then it developed a new refocus algorithm to render picture based on the depth map extracted from the light field data. Comparison of rendering results between this two techniques was also made both for digital refocusing image and all-in-focus image. It can be seen that new algorithm reconstructs result as high resolution as the old algorithm, with artifacts to an unconspicuous level, at the cost of the calculation of depth map estimation. The rendered image has smooth transition at the edge of stitching patches.

\section{Acknowledgment}

The research work was supported by State Key Laboratory of Wireless Mobile Communications, China Academy of Telecommunications Technology (CATT).

\section{References}

[1] Adelson,T. and Wang. Single lens stereo with a plenoptic camera. IEEE Trans Pattern Anal Machine Intell, 14:99-106, 1992.

[2] Ng, Levoy M. et al. Light field photography with a hand-held plenoptic camera. CTSR02. California: Stanford University Computer Science. 2005.

[3] Lumsdaine, A. and Georgiev, T. The focused plenoptic camera. International Conference on Computational Photography, April 2009.

[4] Chihchieh Chen, Yichang Lu, Mingshing Su. Light Field Based Digital Refocusing Using a DSLR Camera with a Pinhole Array Mask. ICASSP2010

[5] A. Lumsdaine. T. Georgiev. Full Resolution Light-field Rendering. Teach.rep, Adobe Systems, January 2008.

[6] Lumsdaine, A. and Georgiev, T., The focused plenoptic camera, in International Conference on Computational Photography, April 2009.

[7] Georgiev, T. and Lumsdaine, A., Reducing plenoptic camera artifacts, Comput. Graph. Forum 29(6), 1955-1968

[8] Liu Peng, Liu Dijun. All-in-focus image reconstruction in plenoptic cameras ICIG 2013

[9] Liu Peng, Liu Dijun. High resolution digital refocusing based on focused plenoptic camera. ICCSA 2014

[10] R. Ng, M. Levoy, M. Brdif, G. Duval, M. Horowitz, and P. Hanrahan. Light field photography with a hand-held plenoptic camera. Tech. Rep., 2005.

[11] R. Ng. Digital light field photography. PhD thesis, Stanford,CA, USA, 2006. AdviserPatrick Hanrahan.

[12] Andrew Lumsdaine, LiliLin, Jeremiah Willcock, Yuduo Zhou, Fourier Analysis of the Focused Plenoptic Camera, SPIE-IS\&T/ Vol. 8667,2013 